\title{
Flight Mechanics of Ram-Scram Transition
}

\author{
Derek J. Dalle* and James F. Driscoll ${ }^{\dagger}$ \\ University of Michigan, Ann Arbor, MI 48109
}

\begin{abstract}
Among known air-breathing propulsion systems, the system that can be operated over the widest range of flight Mach numbers is the dual-mode ramjet-scramjet. In such an engine, either subsonic combustion or supersonic combustion can occur in a shared flowpath, and the mode of operation is determined by the flight condition and control inputs. Recent research has indicated that the steadystate thrust of such an engine is discontinuous across the transition from one mode to the other. This work discusses evidence for that discontinuity and discuss conditions and strategies required to maintain control through ram-scram transition.
\end{abstract}

$\begin{array}{ll}a & =\text { acceleration } \\ A & =\text { duct area } \\ c_{p} & =\text { specific heat at constant pressure } \\ C_{L} & =\text { lift coefficient } \\ C_{D} & =\text { drag coefficient } \\ I & =\text { impulse function } \\ K & =\text { drag polar coefficient } \\ m_{f} & =\text { cumulative fuel consumption } \\ \dot{m} & =\text { mass flow } \\ \dot{m}_{f} & =\text { fuel mass flow rate } \\ M & =\text { Mach number } \\ p & =\text { pressure } \\ q_{\infty} & =\text { freestream dynamic pressure }\end{array}$

\section{Nomenclature}

$\begin{array}{ll}Q_{f} & =\text { specific fuel heat release } \\ R & =\text { normalized gas constant } \\ S & =\text { reference area } \\ t & =\text { time } \\ T & =\text { temperature } \\ u & =\text { total flow speed } \\ \mathbf{u} & =\text { vector of control variables } \\ V & =\text { velocity of vehicle } \\ \mathbf{x} & =\text { vector of state variables } \\ \gamma & =\text { ratio of specific heats, flight path angle } \\ \eta_{c} & =\text { combustion efficiency } \\ \rho & =\text { density } \\ \tau & =\text { temporal integration variable }\end{array}$

\section{Introduction}

A dual-mode ramjet-scramjet engine has the advantage that it can produce positive thrust for a wide range of flight Mach numbers. The challenge is that the pilot or flight control system does not directly control which mode the engine is in. Instead, the mode is primarily determined by the vehicle speed, altitude, and throttle setting. Because the forces and moments on the vehicle can change abruptly when the engine switches from one mode to the other, understanding and predicting this ram-scram transition boundary is critical for the flight dynamics of air-breathing hypersonic vehicles.

The focus of the present work is to address the flight mechanics of this change in thrust, lift, and aerodynamic moment that occurs at ram-scram transition. It may be the case that there is a trim condition on both sides of transition, but a realistic trajectory cannot use both of them as it would require rapid changes in fuel injection, control surface deflections, and angle of attack. Instead it is assumed that all control variables are smooth functions of time across the transition, and this results in flyable ram-scram transition trajectories.

Although previous studies have not investigated flight dynamics across the ram-scram boundary, Bolender et al. [1, 2] investigated the flight dynamics of inlet unstart, which has many similarities. In the case of unstart, the forces on the vehicle also undergo a rapid change, and the stability properties are also affected. It was found in this case that it was possible to stabilize the vehicle after unstart until the inlet could be restarted.

Compared to inlet unstart, ram-scram transition has the simplifying property that it is primarily deterministic. That is, for a given flight condition and fueling condition, it is possible to determine which mode the vehicle is in. The possibility of hysteresis between ram-to-scram and scram-to-ram transition only complicates this slightly. However, an additional complication for the practical operation of a vehicle through ram-scram transition is that it is difficult to measure in flight how close an engine is to transition. Unstart margin can be measured approximately using wall pressure measurements, but measuring how close to thermal choking a given engine is would require measuring the

${ }^{*}$ Graduate Research Assistant, Department of Aerospace Engineering, AIAA Member, dalle@umich . edu

${ }^{\dagger}$ Professor, Department of Aerospace Engineering, AIAA Fellow, jamesfd@umich . edu 


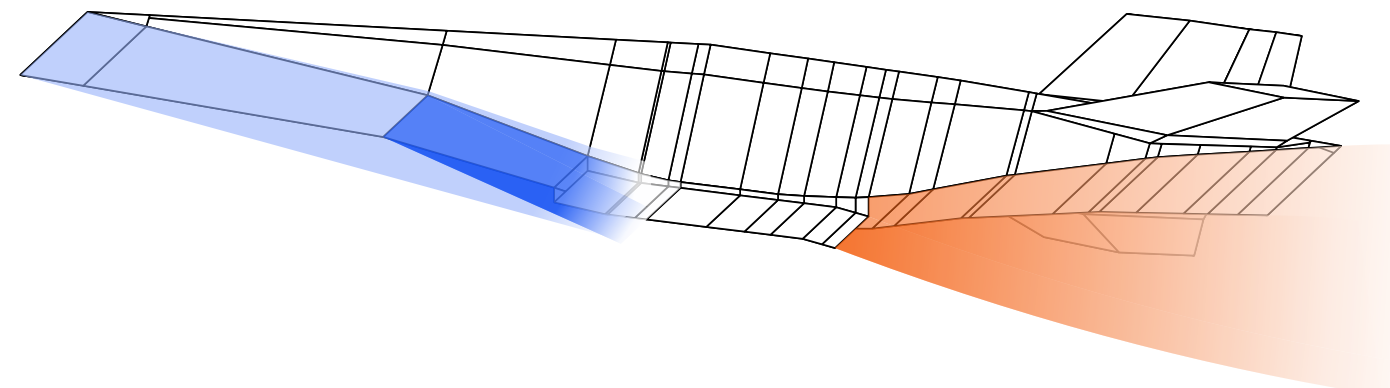

Figure 1. View of MAX-1 vehicle with stylized shocks and exhaust plume.

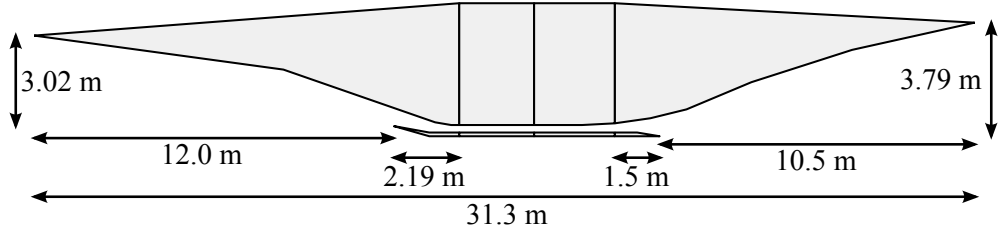

a) Inlet and nozzle dimensions.

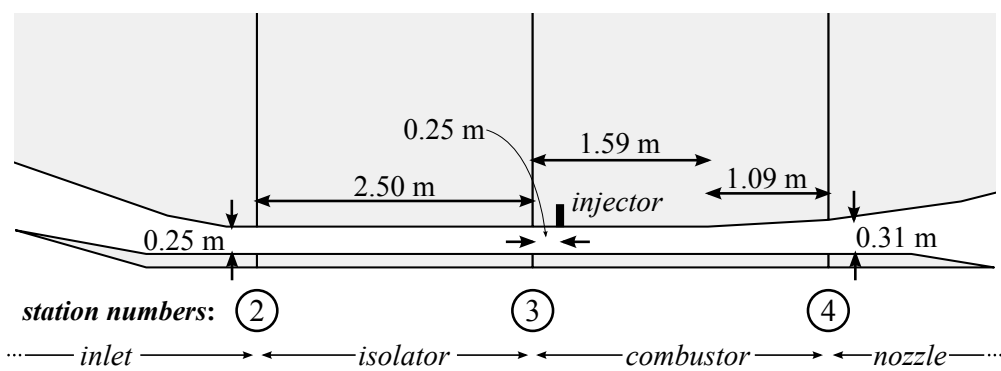

b) Internal flowpath dimensions with station numbers.

Figure 2. MAX-1B engine flowpath dimensions. Engine width is $2.143 \mathrm{~m}$.

Mach number inside the combustor. This emphasizes the importance of calculating transition boundaries preflight.

Many other works have discussed the flight mechanics of scramjet vehicles [3-6] including trajectory analysis [7-10] and feedback control [11,12]. Recent work has also discussed the flight dynamics of both flight modes [13] but not specifically the transition. Chichka et al. [14] and Rodriguez et al. [15] both plotted the thermal choking boundary in terms of flight conditions, but they did not discuss ram-mode flight but rather treated the boundary as an absolute constraint.

In the present work, the final product is a time-domain simulations of stable hypersonic flight through ram-to-scram transition. Although potentially serious consequences are found to be associated with the change in performance at transition, simple strategies to mitigate them are identified and demonstrated. In brief, the flight mechanics of ram-to-scram transition introduce necessary considerations during the vehicle and trajectory design phases, but no insurmountable issues are found.

\section{Dual-Mode Ramjet-Scramjet Vehicle Model}

The MASIV/MASTrim model has been the subject of many previous papers [16-22] and is used for this work. Figure 1 illustrates the generic hypersonic vehicle design used for the present work, and the name "MAX-1B" is associated with this vehicle. The "B" denotes a design with the intention of better performance at the lower Mach numbers in the vicinity of ram-scram transition, whereas the "MAX-1A" design was better suited for pure-scram operation at higher Mach numbers. The propulsive system of the MAX-1B is approximately the same size as the vehicle itself, and can be broken into four components. 


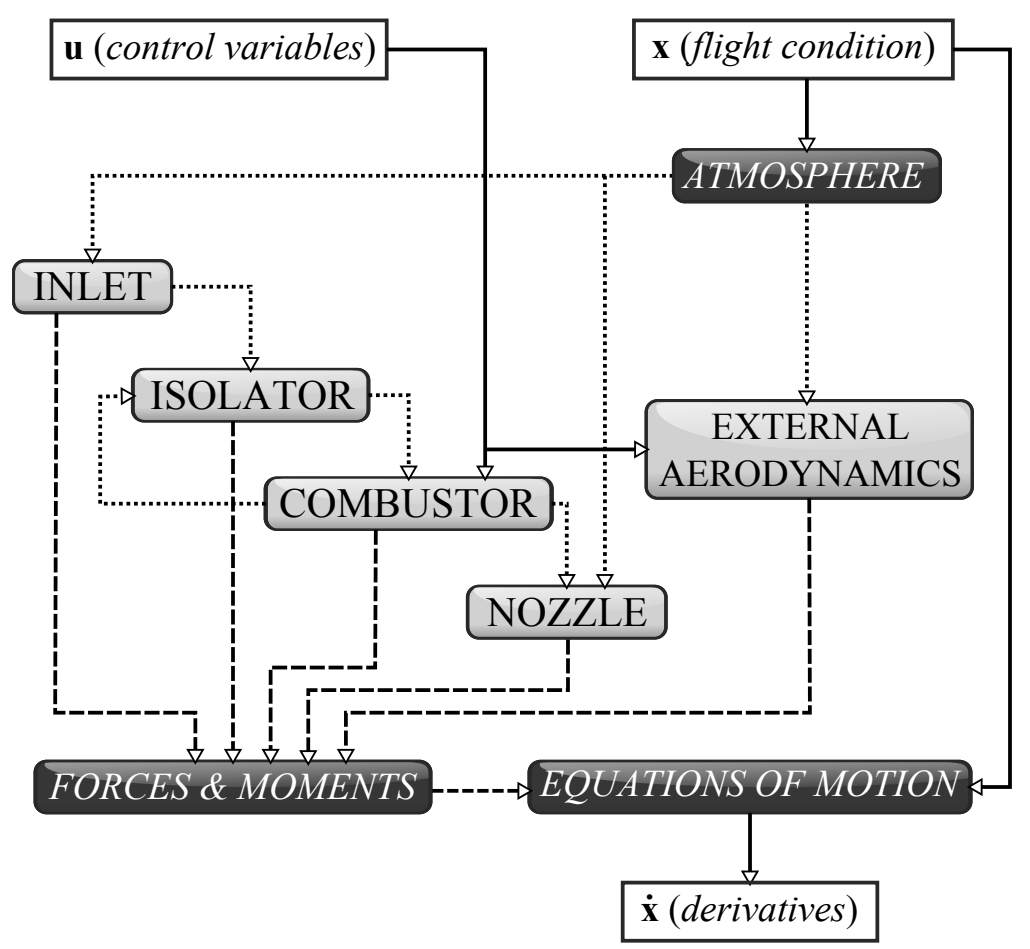

Figure 3. Overview of information flow within the MASTrim program. White blocks represent inputs and outputs, and solid lines show the flow of information to and from these inputs and outputs. Dark blocks represent models not specific to the vehicle, and the gray blocks represent vehicle component models. Dotted lines show information about gas states, and dashed lines show force $\&$ moment information.

An inlet provides both lift for the vehicle to maintain level flight and compression to ensure efficient combustion. This is followed by a constant-area isolator section that separates the inlet from the combustor area where the fuel injection and heat addition occurs. Finally, the exhaust products are allowed to expand in an external nozzle area. These components and the respective dimensions for the MAX-1B vehicle are shown in Fig. 2.

Figure 3 shows how information is passed to and from each component in the MASTrim code. In Fig. 3, there are three types of blocks and three types of arrows, and the information conveyed by these symbols is explained in the caption.

The inputs to the simulation consist of states ( $\mathbf{x}$, also referred to as the flight condition) and a vector of control variables (u). The flight condition is fed into an atmospheric model that determines the freestream air conditions needed for the inlet and external aerodynamics solvers. The four engine components are shown at left in a diagonal pattern. The force and moment created by each of the four engine components is calculated individually, as indicated by the dashed lines in Fig. 3. External pressures and viscous effects naturally provide another source of forces on the vehicle, and this is represented by the fifth input to the forces $\&$ moments block.

Finally, the outputs of the model consist mostly of accelerations and angular accelerations. Calculating these requires more than simply dividing the net forces by the mass or multiplying the inverse of the inertia tensor by the net moments. Instead, the six-degree-of-freedom equations of motion for a rotating ellipsoidal Earth are used.

Figure 4 illustrates the procedure used to model the isolator and combustor flow in cases of ram-mode combustion. The sequence illustrates a case where the engine is in scram mode, and the Mach number remains above unity throughout the combustor (Fig. 4a). The fueling is then increased until the minimum Mach number is exactly 1.0 (Fig. 4b). At this point, it is not possible to add more fuel and keep the engine in scram mode (Fig. 4c). Next, the solver attempts to solve the flow with a maximum-strength shock train (Fig. 4d) and a minimum-strength shock train (Fig. 4e). The true solution will generally be between these two extremes, as shown in Fig. 4f.

\section{Performance Changes at Ram-Scram Transition}

Figure 5 illustrates a control volume in the isolator and combustor during a scram-to-ram transition caused by adding more fuel to a nearly-choked scram condition. It is possible to construct an argument based on a simplified analysis that seems to indicate that transition cannot cause a discontinuity in the thrust between these two conditions. 


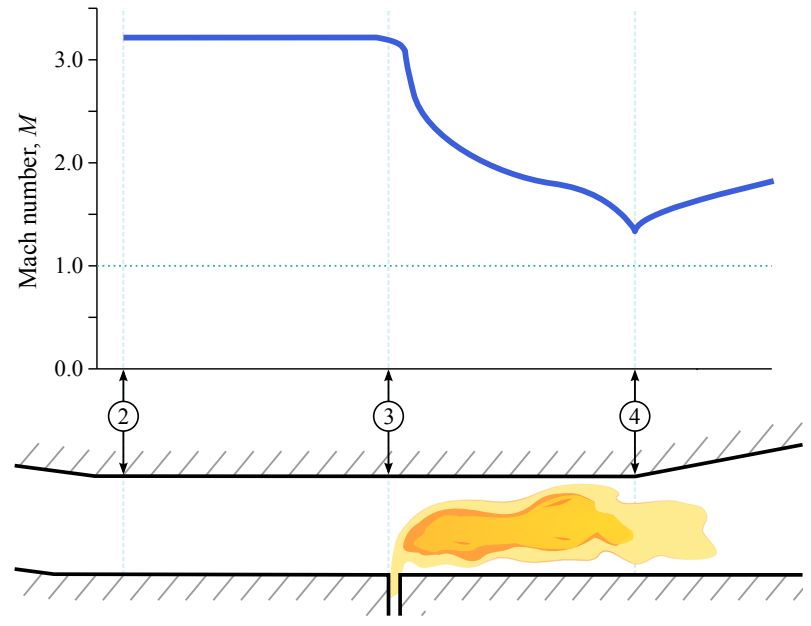

a) Scram mode.

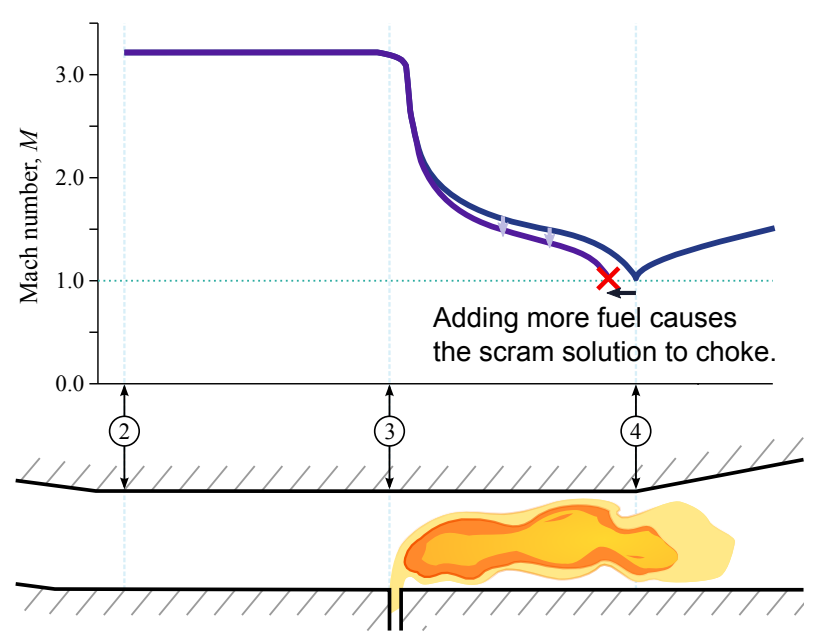

c) Choking.

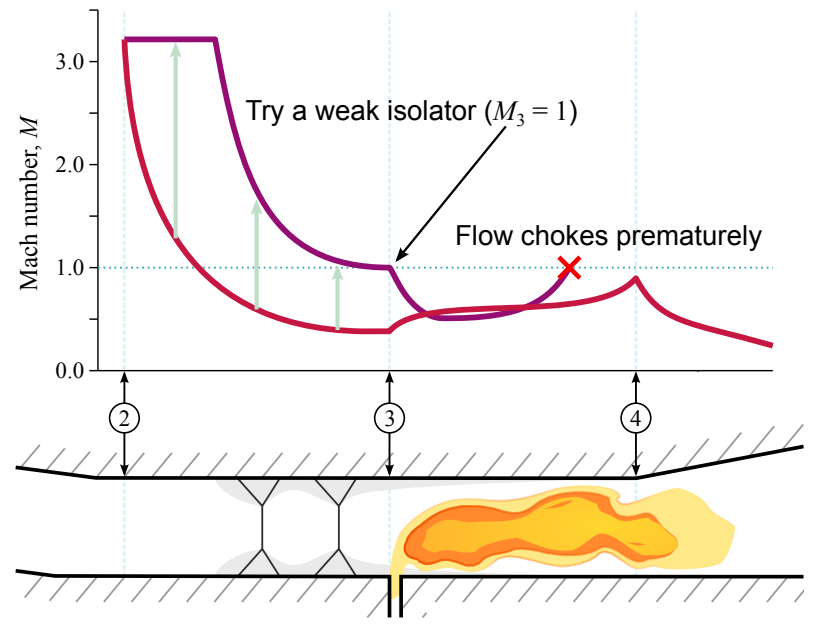

e) Weak shock train.

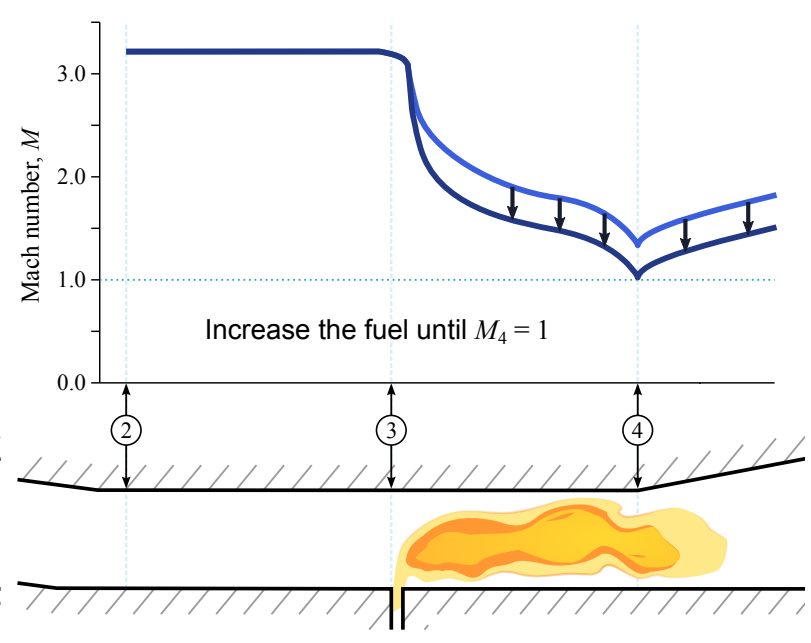

b) Nearly choked.

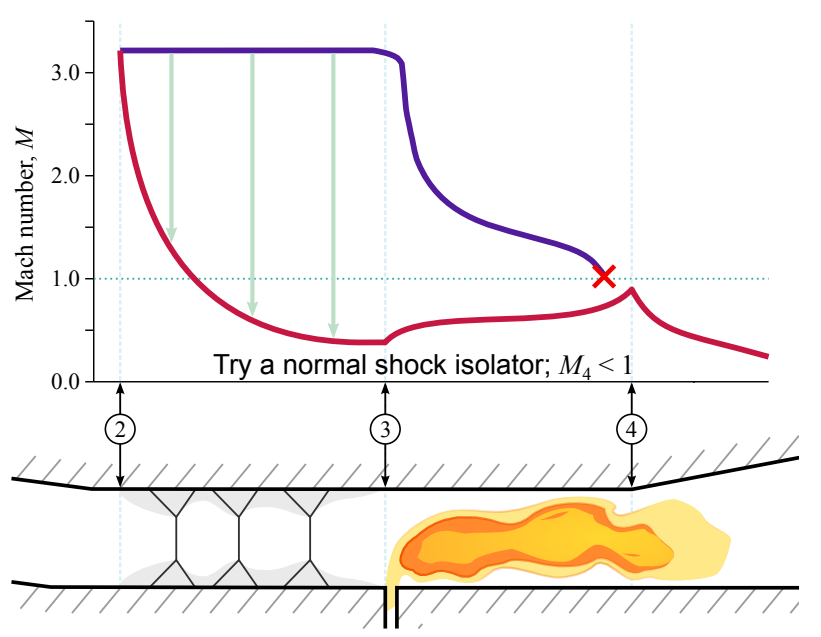

d) Normal-shock isolator.

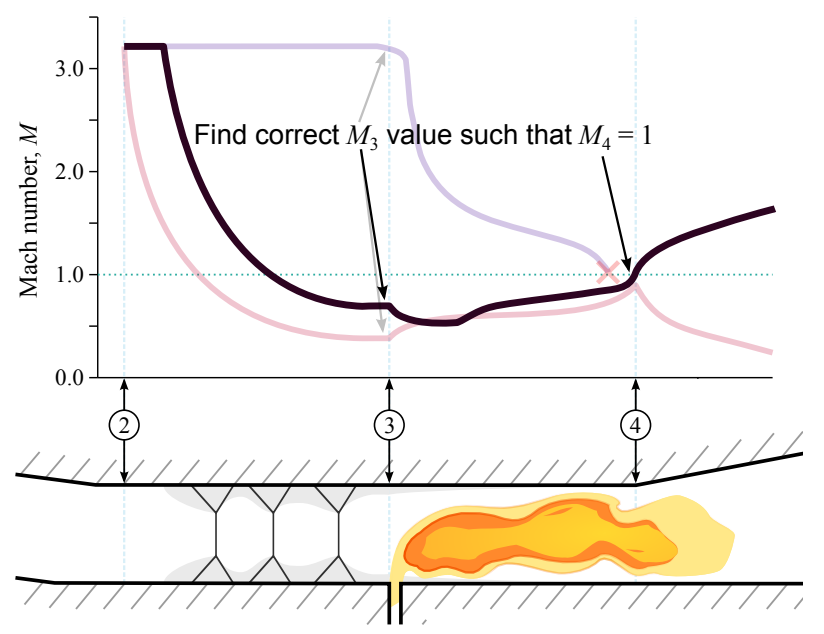

f) Solution.

Figure 4. Diagram of solution method for ram-mode solver. 


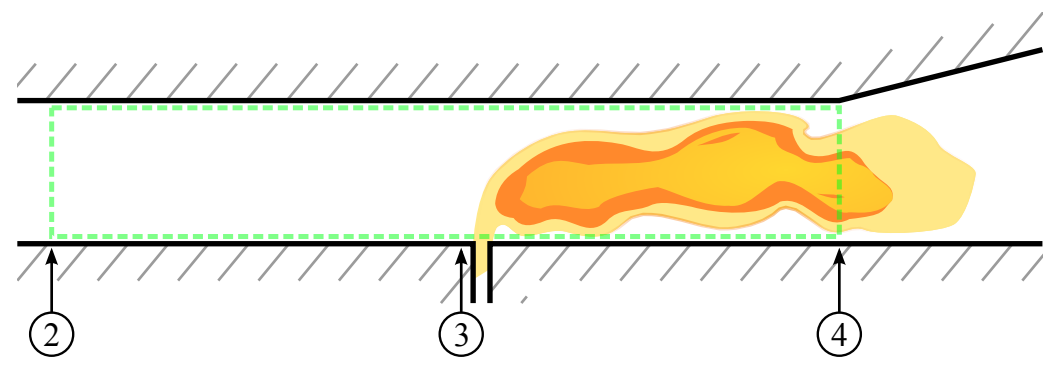

a) Scram mode.

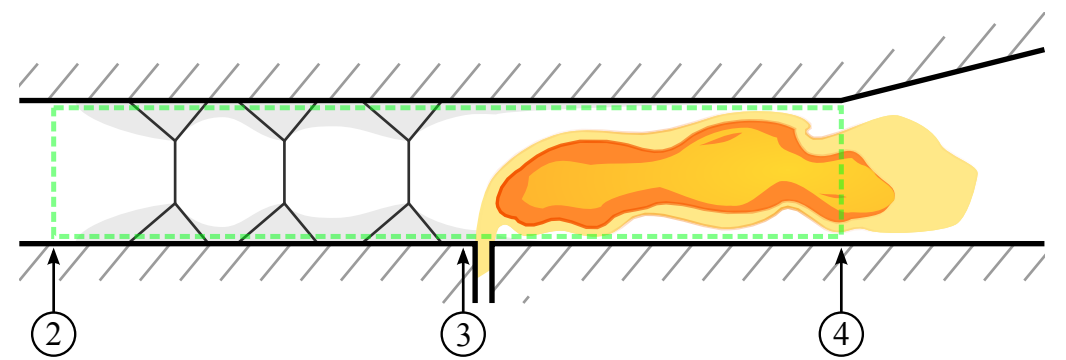

b) Ram mode.

Figure 5. Control volume analysis of conditions immediately before and after scram-to-ram transition.

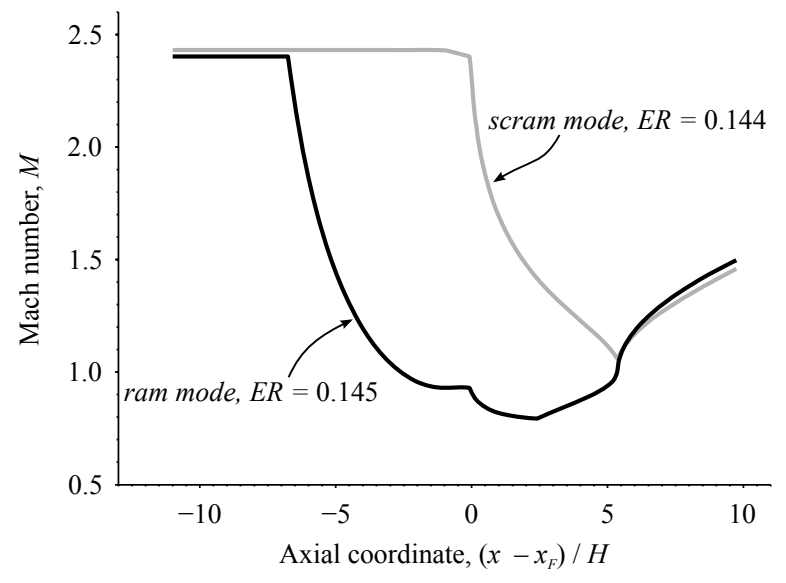

a) Mach number profile.

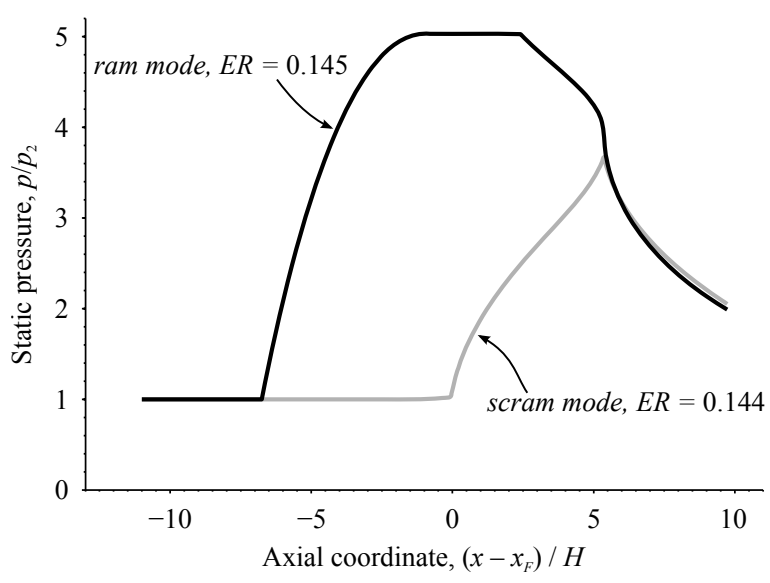

b) Static pressure profile.

Figure 6. Mach number and static pressure profiles for the isolator and combustor of the MAX-1B vehicle under two conditions. The flight Mach number is $M=4.87$, and the dynamic pressure is $100 \mathrm{kPa}$. The acceleration for the ram case is $a=4.44 \mathrm{~m} / \mathrm{s}^{2}$, and for the scram case it is $a=2.15 \mathrm{~m} / \mathrm{s}^{2}$.

In Fig. 5, it is assumed that the transition occurs because of a change in the amount of fuel injected, and the inlet conditions (at station 2) do not change. Then for both cases, the conservation of mass states that

$$
\dot{m}_{4 \mathbf{a}}=\rho_{4 \mathbf{a}} u_{4 \mathbf{a}} A_{4}=\dot{m}_{2}+\dot{m}_{f \mathbf{a}} \quad \dot{m}_{4 \mathbf{b}}=\rho_{4 \mathbf{b}} u_{4 \mathbf{b}} A_{4}=\dot{m}_{2}+\dot{m}_{f \mathbf{b}}
$$

It is assumed that the initial mass flow rate $\left(\dot{m}_{2}\right)$ is the same in both cases, and $\dot{m}_{4 \mathbf{b}}>\dot{m}_{4 \mathbf{a}}$. The conservation of momentum can be written in terms of the impulse function

$$
I_{4 \mathbf{a}}=\left(p_{4 \mathbf{a}}+\rho_{4 \mathbf{a}} u_{4 \mathbf{a}}^{2}\right) A_{4}=I_{2} \quad I_{4 \mathbf{b}}=\left(p_{4 \mathbf{b}}+\rho_{4 \mathbf{b}} u_{4 \mathbf{b}}^{2}\right) A_{4}=I_{2}
$$

Then conservation can be written in terms of the amount of fuel burned:

$$
T_{04 \mathbf{a}}=T_{02}+\frac{\eta_{c \mathbf{a}} Q_{f} \dot{m}_{f \mathbf{a}}}{c_{p} \dot{m}_{2}} \quad T_{04 \mathbf{b}}=T_{02}+\frac{\eta_{c \mathbf{b}} Q_{f} \dot{m}_{f \mathbf{b}}}{c_{p} \dot{m}_{2}}
$$




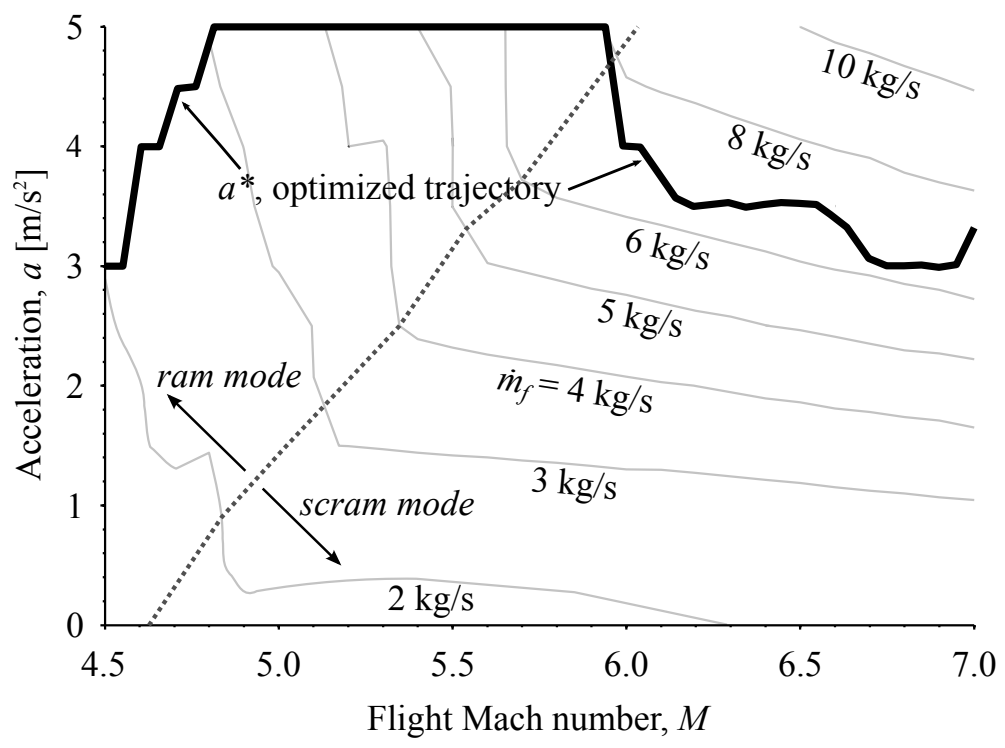

Figure 7. Contours of instantaneous fuel consumption $\left(\dot{m}_{f}\right)$ for MAX-1B vehicle as a function Mach number $(M)$ and acceleration $(a)$ assuming a constant dynamic pressure of $100 \mathrm{kPa}$.

Here $\eta_{c}$ is a combustion efficiency, and $Q_{f}$ is the heat released by burning a kilogram of fuel. It is possible to solve these equations for $M_{4}$, the Mach number at the end of the combustor.

$$
\frac{\left(1+\frac{\gamma-1}{2} M_{4 \mathbf{a}}^{2}\right) M_{4 \mathbf{a}}^{2}}{\left(1+\gamma M_{4 \mathbf{a}}^{2}\right)^{2}}=\frac{R T_{04 \mathbf{a}} \dot{m}_{4 \mathbf{a}}^{2}}{\gamma I_{4 \mathbf{a}}^{2}} \quad \frac{\left(1+\frac{\gamma-1}{2} M_{4 \mathbf{b}}^{2}\right) M_{4 \mathbf{b}}^{2}}{\left(1+\gamma M_{4 \mathbf{b}}^{2}\right)^{2}}=\frac{R T_{04 \mathbf{b}} \dot{m}_{4 \mathbf{b}}^{2}}{\gamma I_{4 \mathbf{b}}^{2}}
$$

Because all of the terms in Eqs. (1)-(4) are smooth functions of $\dot{m}_{f}$, it does not seem that a small change in $\dot{m}_{f}$ can cause a sudden change in the state at station 4. Experience has shown, however, that the combustion efficiency is significantly higher in ram mode than it is in scram mode. This is not surprising as the subsonic flow allows for better fuel-air mixing and longer residence time in the combustor. Thus the main reason for the higher ram-mode thrust compared to scram-mode in the MASIV model is simply that more of the fuel burns in ram mode. Fortunately, the change in moment is much smaller, causing a nose-down angular acceleration of $8 \% \mathrm{~s}^{2}$.

Additionally, there is a small contribution due to friction. In Eq. (2), it is assumed that there is no friction. When friction is considered, however, one notes that the loss of momentum is higher in the scram mode due to (a) higher Reynolds numbers and (b) the fact that there is a separated boundary layer for part of the flow in ram mode. This difference in viscous forces causes a small difference in thrust (about 1 to 5\%), but including it turns out to be a necessary aspect of an engine model because there are no ram-mode solutions without it.

Figure 6 shows an example of a MASIV solution for conditions immediately on either side of ram-scram transition. It can be seen that a very small change in equivalence ratio, which sets how much fuel is injected into the combustor, causes a large change in the pressure and Mach number throughout the isolator and combustor. Although these changes are dramatic, they do not directly affect the forces on the vehicle, because the pressures act on both a top and bottom surface. However, the difference in exit conditions, which is easier to identify in Fig. 6a, does cause a change in thrust. In this case there was a $16 \%$ decrease in thrust, with a net acceleration of $4.44 \mathrm{~m} / \mathrm{s}^{2}$ in ram mode and $2.15 \mathrm{~m} / \mathrm{s}^{2}$ in scram mode.

\section{Operating Maps for the MAX-1B Vehicle}

Figure 7 is an operating map for the MAX-1B vehicle showing fuel consumption required to maintain a constant dynamic pressure and a certain acceleration for Mach numbers between 4.5 and 7.0. It is assumed in all cases that the vehicle is accelerating and climbing along a trajectory with constant dynamic pressure of $100 \mathrm{kPa}$. The boundary between ram mode and scram mode is also shown as a dotted line. A higher acceleration increases the transition Mach number because the thrust required is larger. With the resulting increased equivalence ratios and corresponding heat release, the engine remains in ram mode longer.

Also shown in Fig. (10) is an acceleration profile that minimizes the total fuel consumption to go from Mach 4.5 


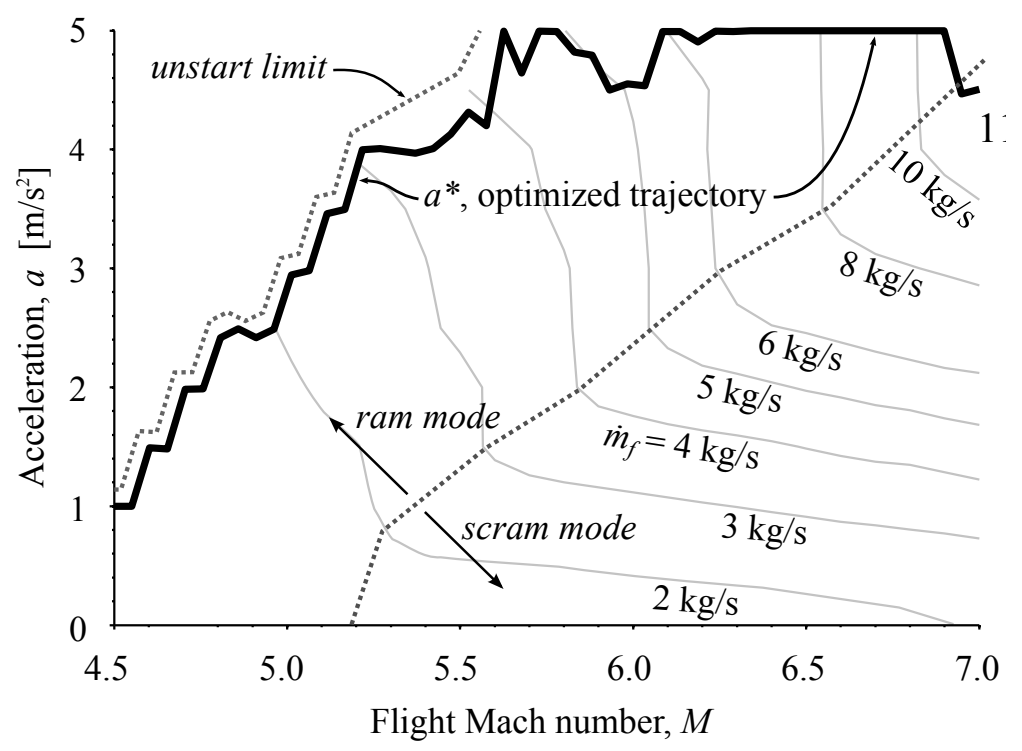

Figure 8. Contours of instantaneous fuel consumption $\left(\dot{m}_{f}\right)$ for MAX-1B vehicle as a function Mach number $(M)$ and acceleration $(a)$ assuming a constant dynamic pressure of $55 \mathrm{kPa}$.

to Mach 7. The total fuel consumption up to a time $t$ can be written

$$
m_{f}(t)=\int_{0}^{t} \dot{m}_{f}\left(V(\tau), a(\tau), m_{f}(\tau)\right) \mathrm{d} \tau
$$

One way to evaluate the fuel consumption is to apply Eq. (5) directly and stop when the velocity reaches the final value. The inconvenience of this approach is that we do not know beforehand how the final value of $t$. A modified approach is to change the variable of integration to be velocity, resulting in

$$
m_{f}\left(V_{N}\right)=\int_{V_{0}}^{V_{N}} \frac{\dot{m}_{f}\left(V, a(V), m_{f}(V)\right)}{V} \mathrm{~d} V
$$

This is more convenient because the acceleration can be scheduled as a function of the velocity (which can easily be converted to Mach number). This leads to a simpler optimization problem in which the decision variables are accelerations $a_{0}, \ldots, a_{N}$ at discrete velocities $V_{0}, \ldots, V_{N}$ distributed throughout the trajectory.

To investigate the effect of altitude on both performance and the location of ram-scram transition, Fig. 8 shows an operating map for the MAX-1B vehicle flying at a constant dynamic pressure of $55 \mathrm{kPa}$. This is much like Fig. 7 except that the altitude is increased. Because the weight of the vehicle is assumed to be constant, flying at a higher altitude (lower dynamic pressure) requires a higher lift coefficient. Because the drag coefficient is approximately a quadratic function of lift coefficient,

$$
C_{D}=C_{D_{0}}+K C_{L}^{2}
$$

flying at a lower dynamic pressure $\left(q_{\infty}\right)$ actually causes the drag to increase. If $S$ is the reference area of the vehicle, and $L$ is constant,

$$
D=q_{\infty} S C_{D_{0}}+\frac{K L^{2}}{q_{\infty} S}
$$

This result is that more fuel is required to maintain the net acceleration, and this tends to keep the vehicle in ram mode to higher Mach numbers.

Due to the higher thrust attained in ram mode, the effects of flying at a higher altitude can be counterintuitive. Normally an air-breathing propulsion system prefers to fly at the lowest altitude allowed by structural or thermal constraints. However, by flying at a higher altitude, the engine can remain in ram mode longer, and the performance increase associated with ram mode may be sufficient to offset the increased drag.

Indeed, Fig. 9 shows that an optimized trajectory prefers a higher altitude in order to remain in ram mode, and this is followed by a dive to the maximum dynamic pressure once the transition to scram mode has been made. Two trajectories are shown: in the first case $(A)$, the trajectory optimizer was free to select any altitude at each Mach number 


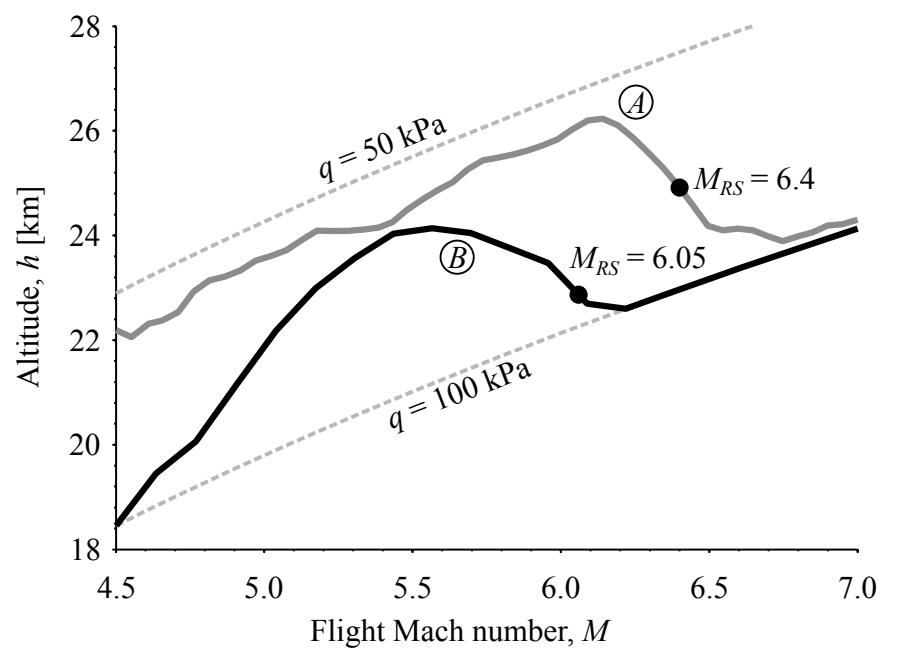

Figure 9. Altitude of optimized trajectory assuming $(A)$ unconstrained initial and final altitude and $(B)$ initial and final dynamic pressure of $100 \mathrm{kPa}$.

as long as the dynamic pressure was between 50 and $100 \mathrm{kPa}$. In the second case, both the initial and final altitudes were constrained, so that the problem describes the possibly more realistic case of a point-to-point trajectory, although the start and end points are in velocity-altitude space rather than corresponding to two points in physical space. The total fuel consumption for the four trajectories are listed below.

- Constant dynamic pressure, $100 \mathrm{kPa}$, optimized acceleration: $1019 \mathrm{~kg}$

- Constant dynamic pressure, $55 \mathrm{kPa}$, optimized acceleration: $898 \mathrm{~kg}$

- Optimized altitude and acceleration: $826 \mathrm{~kg}$

- Optimized altitude and acceleration, initial and final altitudes constrained: $936 \mathrm{~kg}$

In the following sections, simpler trajectories will be analyzed that attempt to fly a constant acceleration through the ram-to-scram transition.

\section{Mach Number Dynamics}

As discussed in Sec. III, there is a significant loss of thrust associated with transition from ram mode to scram mode. Although the present model, which is quasi-steady, does not predict how rapidly this change in performance occurs, it is possible to analyze the effects of this "jump" using conservative assumptions. If the transition is assumed to occur infinitely fast, there will be a sudden drop in acceleration, and this will be accompanied by a net nose-up or nose-down moment. The reason is that neither the equivalence ratio nor the elevator angle can be changed rapidly enough to counteract the change in the vehicle's performance. In this section, we focus on the consequences of this phenomenon on the velocity dynamics. Although the change in acceleration at ram-to-scram transition is significant, the consequences are shown to be acceptable under most circumstances. However, an attempt to cross ram-to-scram transition slowly (that is, with a low ram-mode acceleration), a phenomenon can occur where the net acceleration in scram mode just after transition is negative, causing a reversion to ram mode. The recommendation, then, is to make sure that the acceleration is at least $2 \mathrm{~m} / \mathrm{s}^{2}$ prior to acceleration, or alternatively to make the transition during a dive.

Figure 10 shows a compact summary of the acceleration dynamics for the MAX-1B vehicle. Each light red curve shows the net acceleration as a function of equivalence ratio for a fixed Mach number. The angle of attack and elevator angle are selected so that the vehicle is trimmed to a constant dynamic pressure with no net pitching moment. At each Mach number, a transition occurs at a certain equivalence ratio, which is visible in Fig. 10 as a vertical segment of the red curves.

The vertical segments of the light red curves in Fig. 10 are drawn so that the acceleration vs. equivalence curves are connected, but in actuality they represent a range of net accelerations that cannot be attained at that Mach number. For example, at Mach 4.81, there is no equivalence ratio that gives an acceleration between $0.5 \mathrm{~m} / \mathrm{s}^{2}$ and $3.5 \mathrm{~m} / \mathrm{s}^{2}$. The black curve in Fig. 10 shows the trajectory produced when an automatic trim routine is asked to calculate a trajectory 


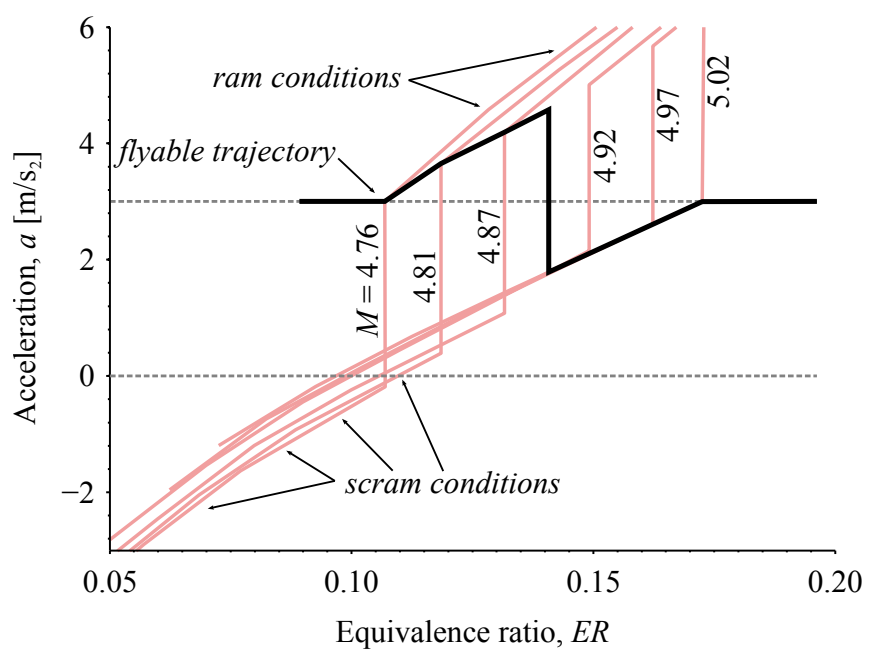

Figure 10. Acceleration as a function of equivalence ratio for increasing Mach number. Between Mach 4.76 and 5.02 there is no equivalence ratio that results in a net acceleration of $3 \mathrm{~m} / \mathrm{s}^{2}$.

with a constant acceleration of $3 \mathrm{~m} / \mathrm{s}^{2}$ from Mach 4.5 to Mach 7. If this curve were shown in isolation, it would appear to show a failure of the trim routine, because the acceleration is clearly not constant. Figure 10 clarifies the issue and demonstrates that it is not possible to attain an acceleration of $3 \mathrm{~m} / \mathrm{s}^{2}$ between Mach 4.76 and Mach 5.02.

The trimmer selected a trajectory that increases the acceleration above $3 \mathrm{~m} / \mathrm{s}^{2}$ prior to ram-scram transition and allows it to drop below in scram mode. This behavior appears somewhat random, but the explanation is relatively simple. Since the trim routine was not able to satisfy the target acceleration, it chose the value that minimized the residual. Further inspection shows that there are several strategies to deal with the inability to maintain a constant acceleration including, but not limited to

1. immediately allowing transition to scram mode once the constant acceleration can no longer be maintained;

2. choosing the acceleration closest to the requested value; and

3. remaining in ram mode until the scram acceleration is $3 \mathrm{~m} / \mathrm{s}^{2}$.

Of these, option 2 is the one shown in Fig. 10. Option 1, which effectively treats $3 \mathrm{~m} / \mathrm{s}^{2}$ as a maximum acceleration, can be seen to have a serious problem. Namely, the maximum scram-mode acceleration at Mach 4.76 is actually negative, and as a result, the trajectory will become stuck in the following cycle.

1. Vehicle accelerates to Mach 4.76 in ram mode.

2. Vehicle switches to scram mode.

3. Net acceleration is negative, so Mach number decreases.

4. Vehicle switches to a ram-mode condition to maintain $3 \mathrm{~m} / \mathrm{s}^{2}$ acceleration.

5. Repeat.

Despite this potentially troublesome oscillation, most of the consequences of the drop in thrust appear to be solvable. For instance, the trajectory shown in Fig. 10 shows that the equivalence ratio is smoothly increasing without any jumps. This is good news for the control design as it negates any need to quickly change the fueling condition at the transition point. In addition, it shows that there are several options available at the point of transition that the trajectory designer can select to meet varying mission requirements. Examples of considerations that may affect the decision of how to perform the transition include total fuel consumption, need to avoid unstart, and effect on pitching stability, which is the subject of the next section. 


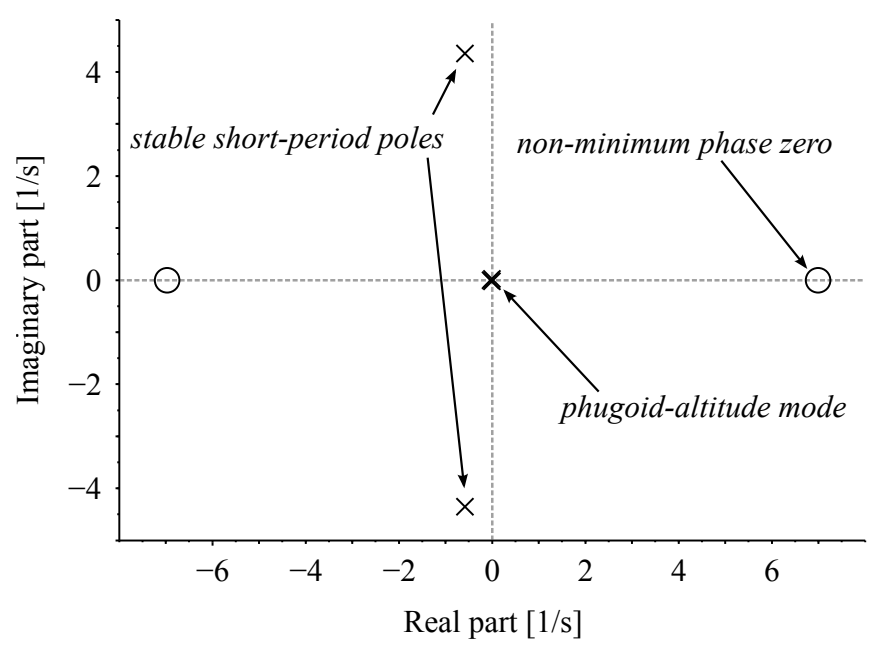

Figure 11. Poles and zeros of the open-loop linearized dynamics of a trimmed scram flight condition just after ram-to-scram transition.

\section{Time-Domain Simulation}

Although the previously described technique of removing time from the simulation is useful for trajectory optimization, understanding the behavior of the vehicle in the immediate vicinity of ram-to-scram transition requires a better understanding of the unsteady behavior. For that reason, the rigid-body dynamics of the MAX-1B vehicle just before and after transition are simulated in this section. It is shown that for the MAX-1B vehicle, the longitudinal dynamics are sufficiently stable that the does not degrade performance significantly even if the transition is assumed to occur infinitely fast.

Figure 11 shows the stability of a Mach 4.92 flight condition trimmed for a constant dynamic pressure of $100 \mathrm{kPa}$. The acceleration is $2.15 \mathrm{~m} / \mathrm{s}^{2}$, and this corresponds to the condition just after ram-to-scram transition from Fig. 10. It can be seen that the short-period pitching dynamics are stable for this condition, suggesting that change in pitching moment induced by ram-to-scram transition might not cause too much oscillation even without sophisticated pitch control. In addition, the pitching moment that occurs immediately after transition is a nose-down moment, which is fortunate because the trimmed scram condition has a lower angle of attack than the trimmed ram condition.

To investigate this stability further, a simple open-loop simulation was performed for a trajectory in which ram-toscram transition occurs. The initial condition is the final trimmed ram-mode condition from Fig. 10, and the control inputs, i.e. the equivalence ratio $(E R)$ and elevator angle $\left(\delta_{e}\right)$, are kept constant. The results are shown in Fig. 12.

After $0.4 \mathrm{~s}$, a transition from ram mode to scram mode occurs, and this causes the pitch angle to decrease sharply, but only for a short time. Just over half a second after the transition, the angle of attack has reached its minimum value, and it begins to increase again. The angle of attack results in Fig. 12a show that the angle of attack oscillates for a few seconds, but the oscillations are fairly well damped. In addition, the oscillations are not particularly large. The time history of the pitch angle shown in Fig. 12b show that although the angle of attack is stabilizing, the pitch angle is still decreasing. This shows that the lift is insufficient to maintain steady flight and thus a higher equivalence ratio and/or lower elevator angle is needed.

The simulation used to produce Fig. 12 had the assumption that the equivalence ratio and elevator angle remain constant, and thus the decrease in flight path angle is not cause for serious concern.

\section{Conclusions}

The flight mechanics of an important phase of hypersonic flight is discussed for the first time. Relevant analysis includes thrust predictions in both ram and scram mode, predictions of transition flight conditions throughout the flight envelope, and time-domain simulations of flight before and after transition.

The direction of transition for an ascent trajectory is from ram mode to scram mode, and this transition is associated with a drop in acceleration of about $2 \mathrm{~m} / \mathrm{s}^{2}$. It was shown that approaching transition with a positive acceleration smaller than this results in an oscillation in which the vehicle tries to accelerate when it is in ram mode but then decelerates when it is in scram mode. Because ram-scram transition occurs at lower Mach numbers for lower altitudes, an alternative to high acceleration is to go through ram-scram transition while the vehicle is descending. 


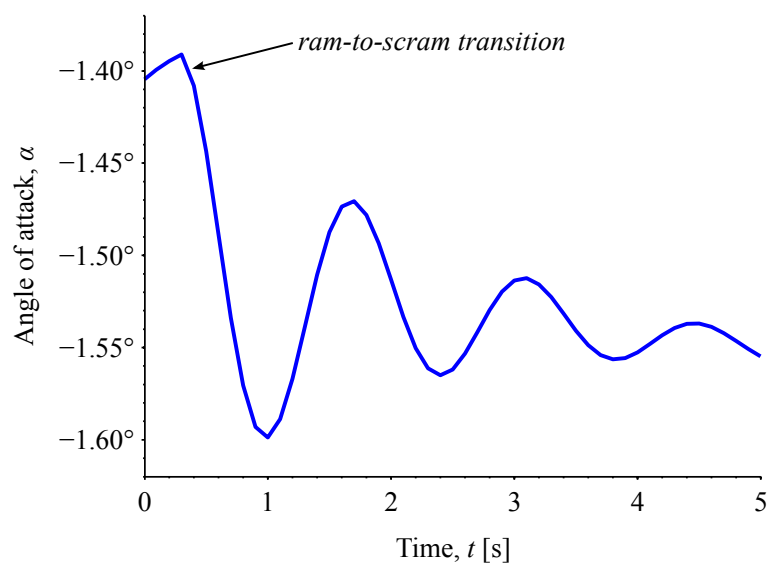

a) Angle of attack $(\alpha)$.

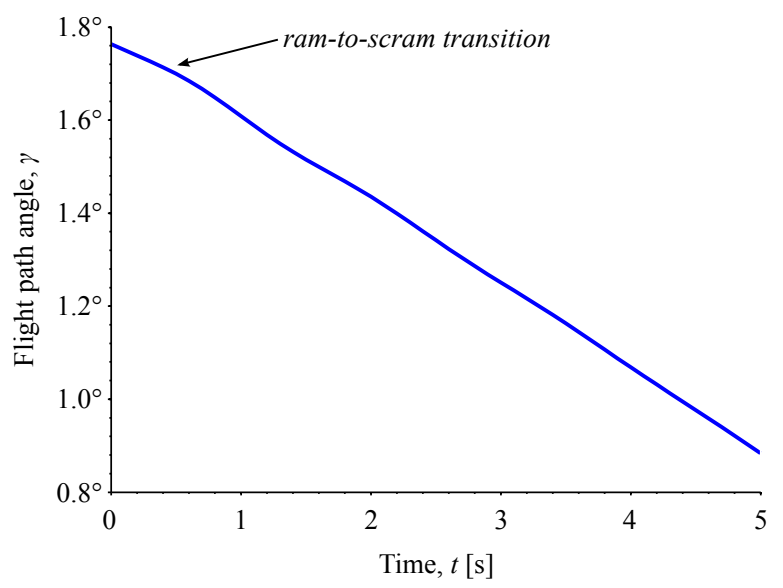

c) Flight path angle $(\gamma)$.

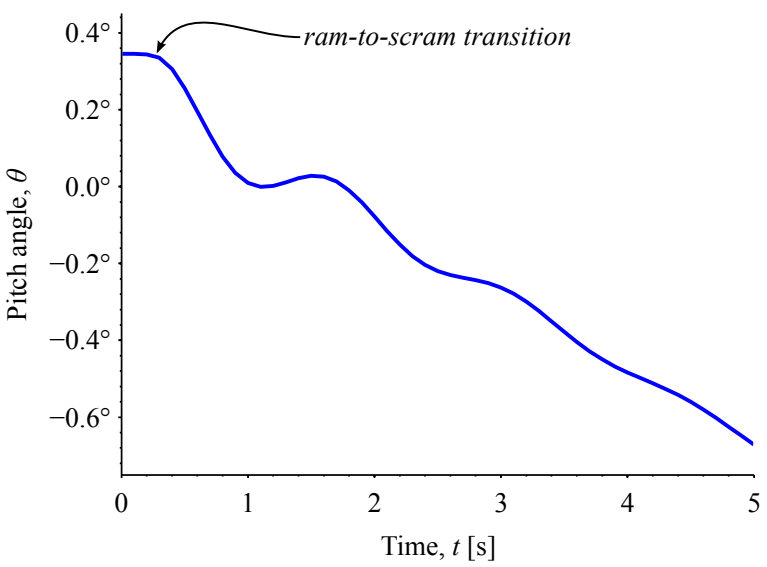

b) Pitch angle $(\theta)$.

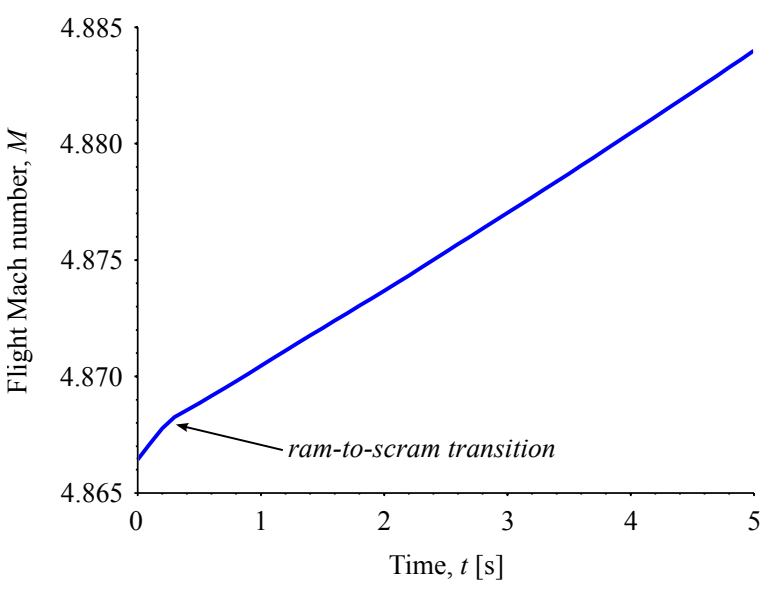

d) Mach number $(M)$.

Figure 12. Open-loop simulation of MAX-1B vehicle with constant equivalence ratio and elevator angle. Initial conditions correspond to a ram-mode condition at an acceleration of $4.5 \mathrm{~m} / \mathrm{s}^{2}$ and a constant dynamic pressure of $100 \mathrm{kPa}$. Ram-to-scram transition occurs at $t=0.4 \mathrm{~s}$.

Although difficulties associated with ram-scram transition were identified, it was shown that they can be overcome without resorting to complex strategies. The MAX-1B vehicle geometry used in this work was longitudinally stable in scram mode, so that the change in pitching moment associated with transition did not cause the angle of attack to diverge. This was the case even if the transition was assumed to occur infinitely fast.

Because ram-scram transition has such an important effect on the flight dynamics of a hypersonic vehicle, it is important to predict the flight conditions and control settings at which it occurs. Trends that tend to cause ram-toscram transition include higher velocities, lower altitudes, and lower acceleration. The opposite trends tend to cause scram-to-ram transition.

\section{Acknowledgements}

This research is funded by the Air Force Research Laboratory/Air Vehicles Directorate grant FA 8650-07-2-3744 for the Michigan/AFRL Collaborative Center in Control Sciences (Michael Bolender as technical monitor).

\section{References}

[1] Bolender, M. A., Wilkin, H., Jacobsen, L., Drayna, T., and Dwenger, A., "Flight Dynamics of a Hypersonic vehicle During Inlet Un-start," 16th AIAA/DLR/DGLR International Space Planes and Hypersonic Systems and Technologies Conference, 2009, AIAA Paper 2009-7292.

[2] Brocanelli, M., Gunbatar, Y., Serrani, A., and Bolender, M. A., "Robust Control for Unstart recovery in Hypersonic Vehicles," AIAA Guidance, Navigation, and Control Conference, 2012, AIAA Paper 2012-4698. 
[3] Chavez, F. R. and Schmidt, D. K., "Analytical Aeropropulsive/Aeroelastic Hypersonic-Vehicle Model with Dynamic Analysis," Journal of Guidance, Control, and Dynamics, Vol. 17, No. 6, 1994, pp. 1308-1319.

[4] Bolender, M. A. and Doman, D. B., "Nonlinear Longitudinal Dynamical Model of an Air-Breathing Hypersonic Vehicle," Journal of Spacecraft and Rockets, Vol. 44, No. 2, 2007, pp. 374-387.

[5] Frendreis, S. G. V., Skujins, T., and Cesnik, C. E. S., "Six-Degree-of-Freedom Simulation of Hypersonic Vehicles," AIAA Atmospheric Flight Mechanics Conference, 2009, AIAA Paper 2009-5601.

[6] Frendreis, S. G. V. and Cesnik, C. E. S., “3D Simulation of a Flexible Hypersonic Vehicle,” Atmospheric Flight Mechanics Conference \& Exhibit, 2010, AIAA Paper 2010-8229.

[7] Calise, A. J., Corban, J. E., and Flandro, G. A., "Trajectory optimization and guidance law development for national aerospace plane applications," American Control Conference, 1988, pp. 1406-1411.

[8] Olds, J. R. and Budianto, I. A., “Constant Dynamic Pressure Trajectory Simulation with POST,” Aerospace Sciences Meeting and Exhibit, 1998, AIAA Paper 1998-0302.

[9] Corban, J. E., Calise, A. J., and Flandro, G. A., "Rapid Near-Optimal Aerospace Plane Trajectory Generation and Guidance," Journal of Guidance, Vol. 14, 1991, pp. 1181-1190.

[10] Lu, P., "Inverse Dynamics Approach to Trajectory Optimization for an Aerospace Plane," Journal of Guidance, Control, and Dynamics, Vol. 16, 1993, pp. 726-732.

[11] Parker, J. T., Serrani, A., Yurkovich, S., Bolender, M. A., and Doman, D. B., "Control-Oriented Modeling of an Air-Breathing Hypersonic Vehicle," Journal of Guidance, Control, and Dynamics, Vol. 30, No. 3, 2007, pp. 856-869.

[12] Schmidt, D., "Integrated Control of Hypersonic Vehicles," AIAA/DGLR Fifth International Aerospace Planes and Hypersonics Technologies Conference, 1993, AIAA Paper 1993-5091.

[13] Dalle, D. J., Torrez, S. M., and Driscoll, J. F., "Unstart Margin and Ram-Scram Transition Boundaries Computed for Hypersonic Vehicles," Journal of Propulsion and Power, 2013, Submitted.

[14] Chichka, D. F., Shankar, U. J., Cliff, E. M., and Kelley, H. J., "Cruise-Dash-Climb Analysis of an Airbreathing Missle," Journal of Guidance, Vol. 11, No. 4, 1988, pp. 293-299.

[15] Rodriguez, A. A., Dickeson, J. J., Cifdaloz, O., McCullen, R., Benavides, J., Sridharan, S., Kelkar, A., Vogel, J. M., and Soloway, D., "Modeling and Control of Scramjet-Powered Hypersonic Vehicles: Challenges, Trends, \& Tradeoffs," AIAA Guidance, Navigation and Control Conference and Exhibit, 2008, AIAA 2008-6793.

[16] Dalle, D. J., Fotia, M. L., and Driscoll, J. F., "Reduced-Order Modeling of Two-Dimensional Supersonic Flows with Applications to Scramjet Inlets," Journal of Propulsion and Power, Vol. 26, No. 3, 2010, pp. 545-555.

[17] Torrez, S. M., Driscoll, J. F., Ihme, M., and Fotia, M. L., "Reduced Order Modeling of Turbulent Reacting Flows With Application to Scramjets," Journal of Propulsion and Power, Vol. 27, No. 2, March-April 2011, pp. 371-382.

[18] Dalle, D. J., Torrez, S. M., and Driscoll, J. F., "Rapid Analysis of Scramjet and Linear Plug Nozzles," Journal of Propulsion and Power, Vol. 28, No. 3, 2012, pp. 545-555.

[19] Dalle, D. J., Torrez, S. M., and Driscoll, J. F., “Turn Performance of an Air-Breathing Hypersonic Vehicle,” AIAA Atmospheric Flight Mechanics Conference, 2011.

[20] Dalle, D. J., Torrez, S. M., Driscoll, J. F., and Bolender, M. A., "Flight Envelope Calculation of a Hypersonic Vehicle Usinga First Principles-Derived Model," 17th AIAA International Space Planes and Hypersonic Systems and Technologies Conference, 2011, AIAA Paper 2011-2394.

[21] Dalle, D. J., Torrez, S. M., and Driscoll, J. F., "Performance Analysis of Variable-Geometry Scramjet Inlets Using a Fundamental Model," 47th AIAA/ASME/SAE/ASEE Joint Propulsion Conference and Exhibit, 2011.

[22] Vick, T. J., Muse, J. A., and Bolender, M. A., "A Hypersonic Vehicle Model Generator for MASIV," AIAA Modeling and Simulation Technologies Conference, 2012. 